ARTIFICIAL SATELLITES, Vol. 54, No. 4 - 2019
DOI: 10.2478/arsa-2019-0009

sciendo

\title{
OBSERVATION OF LEO OBJECTS USING OPTICAL SURVEILLANCE FACILITIES: THE GEOGRAPHIC ASPECT
}

\author{
O.M. Kozhukhov, T.O. Dementiev, S.V. Rischenko \\ The Centre for Special Data Reception and Processing and Navigating Field Control, \\ Zalistsi village, Dunaivtsi district, Khmelnytskyi region, 32444, Ukraine; \\ Tel./fax: +380 (3858) 31 830; e-mail: a.m.kozhukhov@gmail.com \\ N.I. Koshkin, L.S. Shakun, S.L. Strakhova \\ Research Institute Astronomical Observatory of Odessa I.I. Mechnikov National University, \\ Odessa, Ukraine; e-mail: nikkoshkin@yahoo.com \\ O.M. Piskun, D.M. Kozhukhov, O.M. Iluchok \\ National Space Facilities Control and Test Centre, Kyiv, Ukraine \\ S.S. Moskalenko, S.M. Korniyevskiy \\ Ministry of Defense, Kyiv, Ukraine
}

\begin{abstract}
Simulation modelling of the observability of low Earth orbit (LEO) objects was performed using optical surveillance facilities depending on their geographic location and time of year. Orbital data for LEO objects from the open-access catalogue of the near-Earth space objects of the US Combined Space Operations Center (CSpOC) were taken as the initial data for the simulation. The simulation results revealed a complex relationship between the pattern of observability of a LEO object, its orbital parameters and location of the optical surveillance facility, in particular, for Sun-synchronous orbits (SSO) and observing facilities located near the equator. We also discuss variations in the frequency of passes of LEO objects into the field of view (FOV) and in the duration of their observation while passing through the FOV for optical surveillance facilities at three alternative locations. The obtained results and modelling techniques can be further used in the location planning of new optical observing facilities.
\end{abstract}

Keywords: LEO objects, simulation modelling, optical observations, observation scheduling.

\section{INTRODUCTION}

In recent years, there has been growing interest in optical observations of space objects in low Earth orbit (LEO), including space debris. Advances in light-detecting devices have resulted in more frequent usage of optical surveillance facilities (OSF) to observe LEO objects. Not only is the number of optical devices growing, but also they are found to be increasingly more capable; research, development and engineering activities are well underway aiming at making such devices more versatile and useful, as is the case, for instance, for optical sensors for tracking objects in LEO (Lal et al., 2018). Optical sensors are certainly trail the radar ranging systems in respect of the scope of observations and capability to conduct survey irrespective of the weather conditions and the time of day; on the other hand, they have a significant advantage in their 
efficiency in terms of the development and maintenance costs, ensuring at that high accuracy of both estimated positions of LEO objects (Sánchez-Ortiz, 2018; Shakun et al., 2017) and assessment of their current status from the photometric observations (Koshkin et al., 2018; Shakun et al., 2014; Silha et al., 2018) or dynamic parameters (Friedman \& Frueh, 2018). Besides, at relatively low costs (as compared with the costs of radar ranging systems), the OSF enable conducting observations of extremely small LEO objects, such as nanosatellites (Gasdia et al., 2017), using new detectors among others (Schildknecht et al., 2014).

However, the issue of the best OSF location in order to improve the effectiveness of LEO object observations, that is, observability of LEO objects (or visibility at a specific time using a given OSF) is still underexplored. Techniques for simulation modelling of flybys within the OSF field of view (FOV) for a LEO object in an artificially created orbit (Piergentili et al., 2014), as well as for several thousand of real objects over a long period of time (Vananti et al., 2013), have been deployed to deal with the above problem. It is evident that the best results of such experiments can be achieved by performing modelling for the entire population of known LEO objects at once, regardless their considerable number (more than 15,000 registered objects). Also, such a modelling should be conducted simultaneously for several OSF located at different geographic points. However, as far as we know, such surveys are lacking.

\section{TECHNIQUES USED FOR MODELLING THE OBSERVABILITY OF LEO OBJECTS}

Selection of OSF. The following OSF were selected as observing locations for further simulation of LEO object flybys within their FOVs (Figure 1):

- Now operating OSF named Quantum-Optical System “Sazhen-S” of the Centre for Special Data Reception and Processing and Navigating Field Control (it is the branch of National Space Facilities Control and Test Center of the State Space Agency of Ukraine) (hereinafter referred to as OSF-1) with the geographic coordinates $48.85^{\circ} \mathrm{N}, 26.72^{\circ} \mathrm{E}$;

- Assumed OSF (hereinafter referred to as OSF-2), located on the equator: latitude $0.0^{\circ} \mathrm{N}$. In our calculations, we used OSF-2 having the same longitude as OSF-1 equal to $26.72^{\circ} \mathrm{E}$, a longitude shifted at $\approx 90^{\circ}$ from it, and also a longitude of $30.75^{\circ} \mathrm{E}$;

- Assumed OSF (hereinafter referred to as OSF-3) located in Antarctica, at the point corresponding to the coordinates of Ukrainian Antarctic Station "Academician Vernadsky" $\left(65.25^{\circ} \mathrm{S}, 64.26^{\circ} \mathrm{W}\right)$.

It was assumed that each of these OSF has all-round FOV with a minimum elevation above the horizon of $13^{\circ}$. 


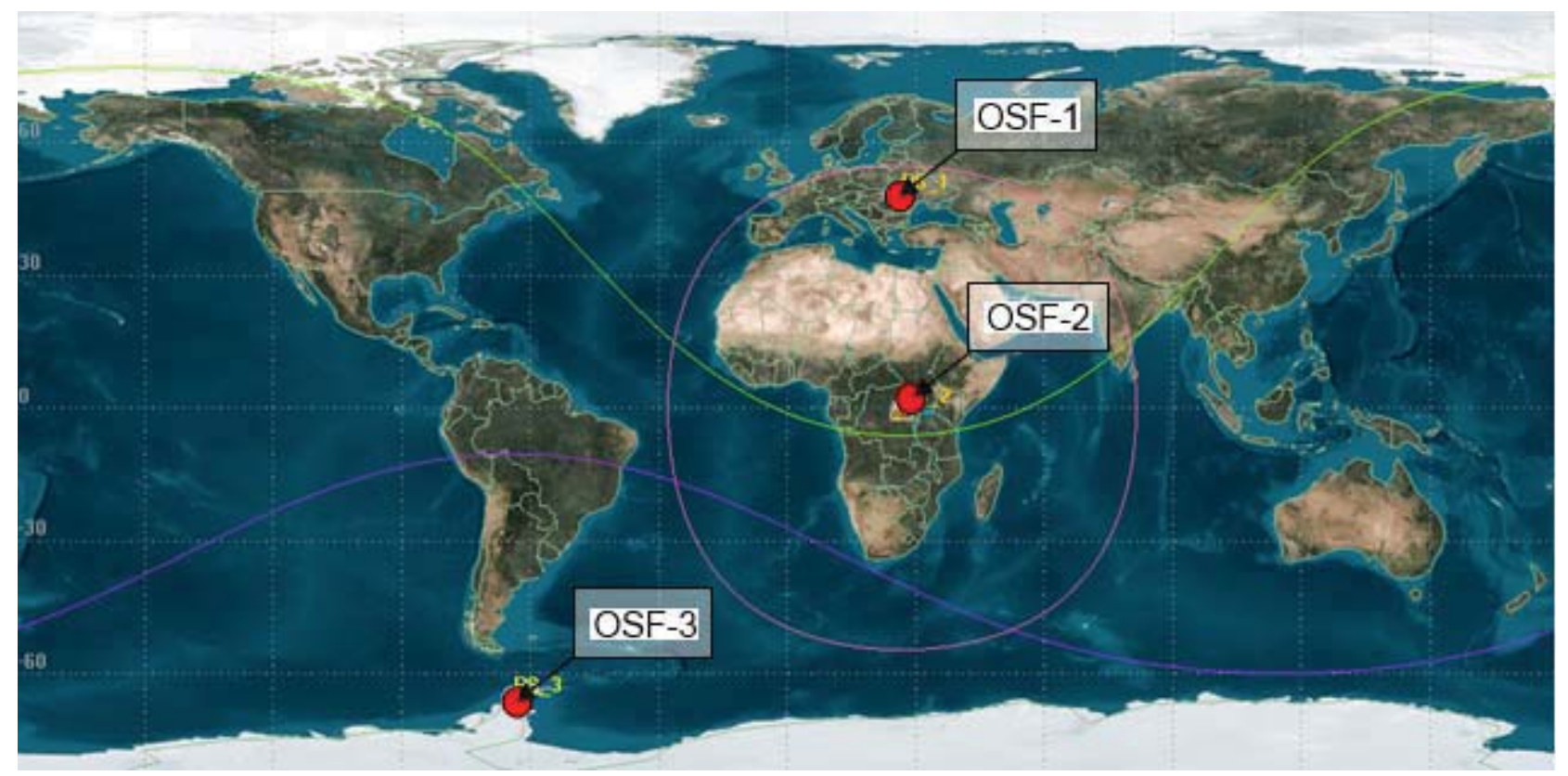

Fig. 1. OSF location and their fields of view

Specific aspects of the simulation. Orbital data as the initial of these for the simulation in the form of two-line element sets as of 13.03.2018 from the open-access catalogue of the U.S Combined Space Operations Center (CSpOC) which is a part of the United States Strategic Command (USStratCom) were taken. Space objects with apogees less than 2,000 km and perigees greater than $140 \mathrm{~km}$, making a total of 11,369 space objects, were selected from the USStratCom catalogue. Formerly it was named the North American Aerospace Defence Command (NORAD) catalogue (see NORAD).

The calculations were carried out separately for two groups of LEO objects: the first group included all LEO objects, while the second one contained only those deemed to be operating spacecrafts in LEO; in our list the latter made a total of 1,062. The passes (revolutions) during which the space objects crossed the OSF FOV were predicted for each OSF and for each group of space objects for a given night at first (as discussed further below). Predictions were made taking into account the lighting conditions, that is, observations could be performed after sunset at the OSF location while the observed space object in orbit should be illuminated by the Sun.

Then, we computed the LEO object paths through the OSF FOV with the time step of 1 second for each of the revolutions predicted as described above. The computations were performed using specially designed software (SDS) of the Ukrainian Outer Space Monitoring Center (OSMC) and adjunct programmes developed by the OSMC and Astronomical Observatory of Odessa National University (AO ONU) specialists. An analytical model of space object motion "SGP4/SDP4" recommended for simulations with the TLE format of the initial data (Hoots et al., 1980) was used in the computations.

\section{ANALYSIS OF THE OUTPUT DATA OF THE LEO OBJECTS OBSERVABILITY SIMULATION}

The number of the LEO objects' passes through the OSF FOV. The passes (flybys) of LEO space objects were predicted for four 1-day intervals in 2018 falling about:

- Vernal equinox: from 20.03.2018, 16:00 UTC to 21.03.2018, 07:00 UTC;

- Summer solstice: from 21.06.2018, 16:00 UTC to 22.06.2018, 07:00 UTC; 
- Autumn equinox: from 23.09.2018, 16:00 UTC to 24.09.2018, 07:00 UTC;

- Winter solstice: from 22.12.2018, 16:00 UTC to 23.12.2018, 07:00 UTC.

The resulting number of the LEO object passes through the FOV of the three indicated OSF is presented in Figures 2 and 3. These calculations bring to one's attention that the number of visible LEO objects, as well as of their passes over the equatorial OSF-2, is noticeably less than the other two, irrespective of the time of year. To verify this result, we carried out an additional model experiment.
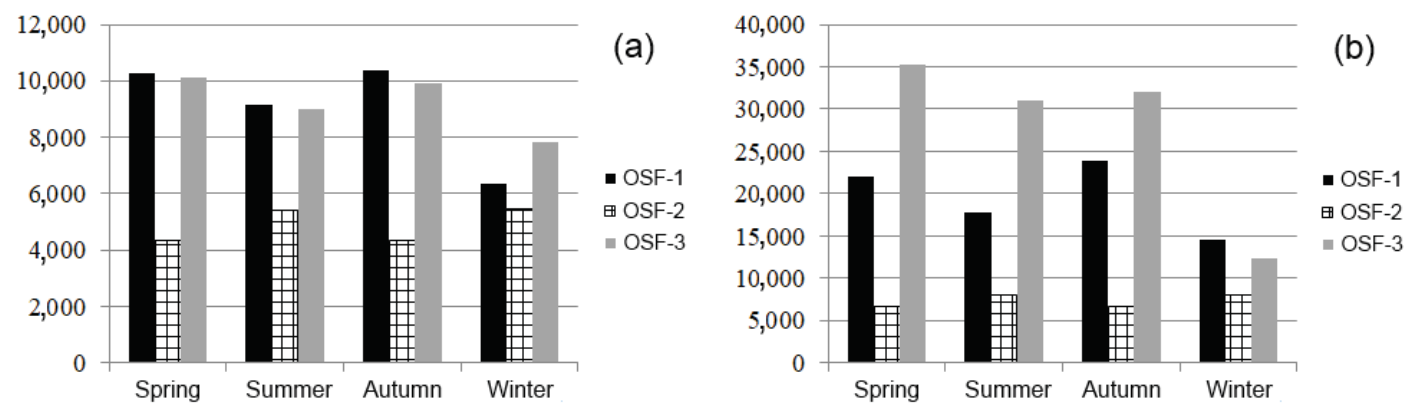

Fig. 2. The LEO object passes through the field of view of three OSF at different times of the year: (a) the number of LEO objects; (b) the number of the LEO object passes
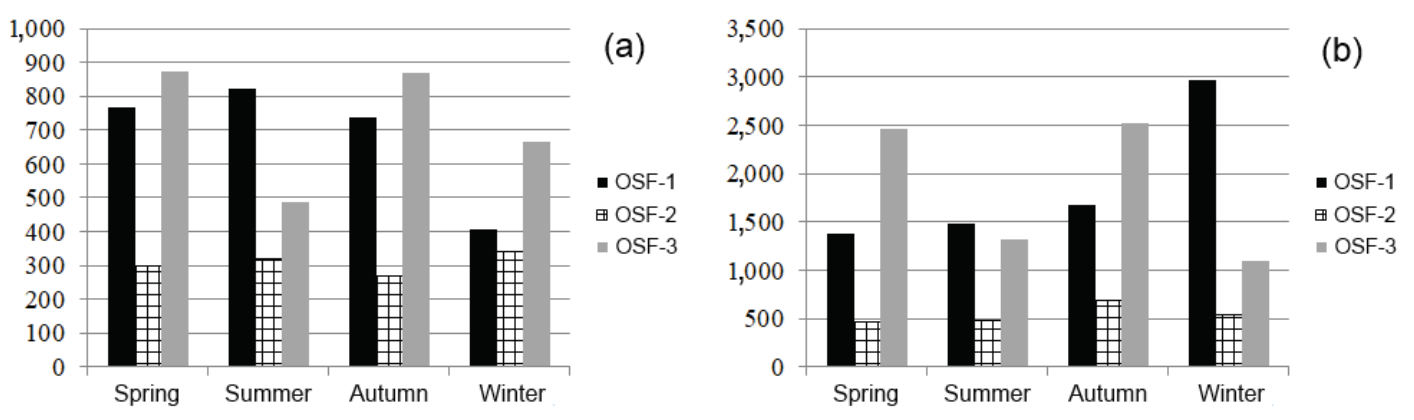

Fig. 3. The LEO spacecraft passes through the field of view of three OSF at different times of the year: (a) the number of LEO spacecrafts; (b) the number of the LEO spacecraft passes

Numerical experiments to determine the number of the LEO objects' passes through the FOV of the equatorial OSF-2. To verify the simulation findings for OSF-2, an additional simulation was carried out for the indicated list of LEO spacecrafts observed at the equatorial OSF-2. The main reason for conducting such a simulation for LEO spacecrafts only is that their number makes a sufficient statistical sample while the relevant calculations take substantially less time than those for the entire quantity of LEO objects.

The predictions of the LEO spacecraft flybys were made for three equatorial OSF on the nights of the 1st/2nd (indices 1-3) and 15th/16th (indices 1a-3a) of each calendar month throughout 2018. The results obtained are illustrated in Figure 4. On the whole, the results of the additional simulation confirmed the earlier described simulation findings for OSF-2. The number of LEO spacecrafts observed at three equatorial OSF throughout the year varies from 255 to 340, whereas the number of visible passes ranges from 390 to 550. Thus, the estimations made on the basis of just four short intervals during the year were confirmed (Figure 3).

We supposed that objects in Sun-synchronous orbits (SSO) making up a substantial proportion of the total list of LEO objects, as well as specific features of their illumination, contributed to such statistics. 

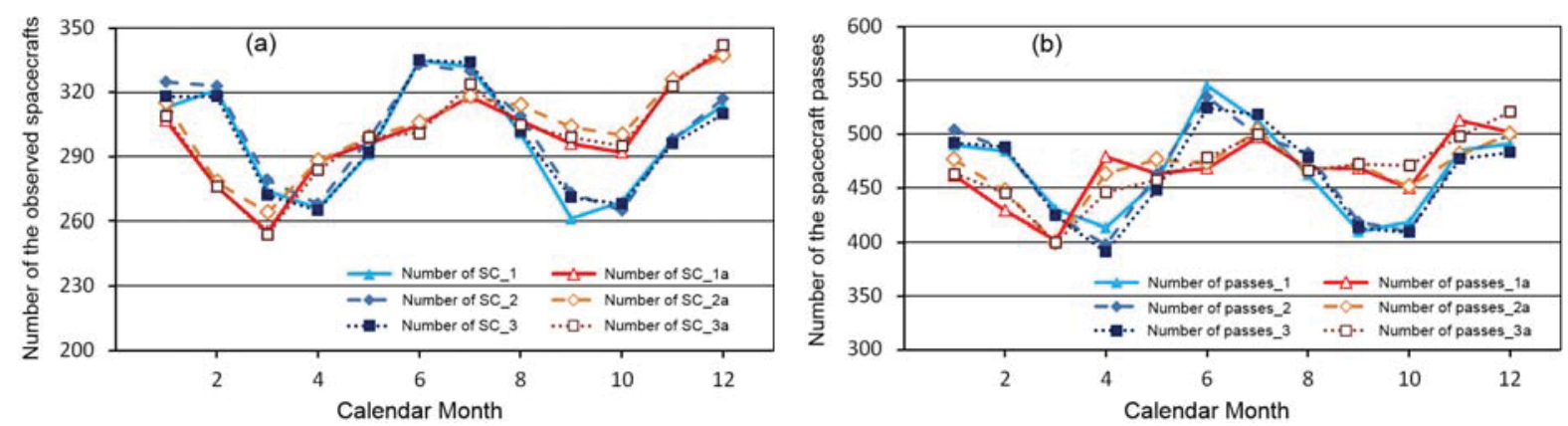

Fig. 4. (a) The number of LEO spacecrafts visible during a single night throughout the year 2018 (either of nights of the 1st/2nd and 15th/16th of each calendar month, which are depicted separately with solid and open markers, respectively) at three equatorial OSF (with longitudes $26.72^{\circ} \mathrm{E}, 116.72^{\circ} \mathrm{E}$ and $64.72^{\circ} \mathrm{W}$ ); (b) the respective number of visible passes of those LEO spacecrafts

Testing observability of LEO spacecrafts in SSO for the equatorial OSF. The computations were performed for the equatorial OSF with $30.75^{\circ} \mathrm{E}$ longitude. At first, to carry out the experiment, we selected 11 LEO spacecrafts in near-circular orbits with inclinations ranging from $97.57^{\circ}$ to $99.27^{\circ}$ and a mean motion of 13.43-15.47 revolutions per day (Table 1). Due to the orbital precession, the right ascension of the ascending node (RAAN) of SSO increases at a rate similar to that of the annual increase of the Sun's equatorial coordinate. Furthermore, two spacecrafts of the German satellite system SAR-Lupe (International Identification Numbers are 07053A and 08036A) and Ukrainian spacecraft BPA-3/SL-24 (Block of Promising Avionics mounted to the third stage of the Dnepr Rocket, 13066AJ), have a noticeable deviation of the rate at which their orbital planes shift with respect to the Sun, that is, they are not spacecrafts in SSO.

Table 1. Space objects in low Earth orbits close to the Sun-synchronous ones, selected for the simulation of the observability at the equatorial OSF.

\begin{tabular}{|c|l|}
\hline $\begin{array}{c}\text { Reference no. } \\
\text { in the CSpOC/USStratCom } \\
\text { catalogue }\end{array}$ & $\begin{array}{c}\text { Name of the LEO object } \\
\text { (International Identification } \\
\text { Number) }\end{array}$ \\
\hline 27421 & SPOT-5 (02021A) \\
\hline 28654 & NOAA-18 (05018A) \\
\hline 32283 & SAR-LUPE 3 (07053A) \\
\hline 33244 & SAR-LUPE 5 (08036A) \\
\hline 33591 & NOAA-19 (09005A) \\
\hline 37387 & RESOURCESAT-2 (11015A) \\
\hline 38038 & ZY-1 (11079A) \\
\hline 38710 & TET-1 (12039D) \\
\hline 39448 & BPA-3/SL-24 (13066AJ) \\
\hline 40699 & COSMOS-2506 (15029A) \\
\hline 41579 & COSMOS-2517 (16034A) \\
\hline
\end{tabular}

As regards the other eight spacecrafts, they show a nodal precession rate close to the Sun's angular velocity, but their orbital planes are at different angular distances from the Sun along the celestial equator. We will further consider angle $\mathrm{D}$ as the difference between the RAAN of the satellite's orbit and the relevant right ascension of the Sun: D = RAAN ${ }_{\text {Sat }}-$ RAsun. Figure 5 shows plotted variations of the RAAN of the given spacecrafts during the period from March to 
November 2017. Variations in the right ascension of the Sun over the specified interval are illustrated at the bottom of the plot.

The observability of the given LEO spacecrafts at the equatorial OSF during 2017 was inferred by direct computation of the ephemerides with standard software, which is usually deployed to schedule and perform observations of LEO objects at AO ONU (Shakun et al., 2014, 2016, 2017). Orbital elements of LEO spacecrafts were updated fortnightly (if they were available online in the CSpOC/USStratCom catalogue or on the Mike McCants Satellite Tracking web pages [see Mike McCants' website]). Figure 6 (upper panel) plots the altitude of the spacecraft Cosmos-2506 (15029A) as a function of time for each of its visible passes during 2017 (each pass is depicted with several altitudes for a given date with maximum values corresponding to the spacecraft culminating above the observer's horizon). The angular distance of the RAAN of this spacecraft from the Sun is about $130^{\circ}$. Due to such an orbital position relative to the Sun, the LEO spacecraft's passes could only be observed at the equatorial OSF during several days in summer 2017 with the altitudes at culmination not higher than $16^{\circ}$ above the horizon and were not visible at all other times throughout the year.

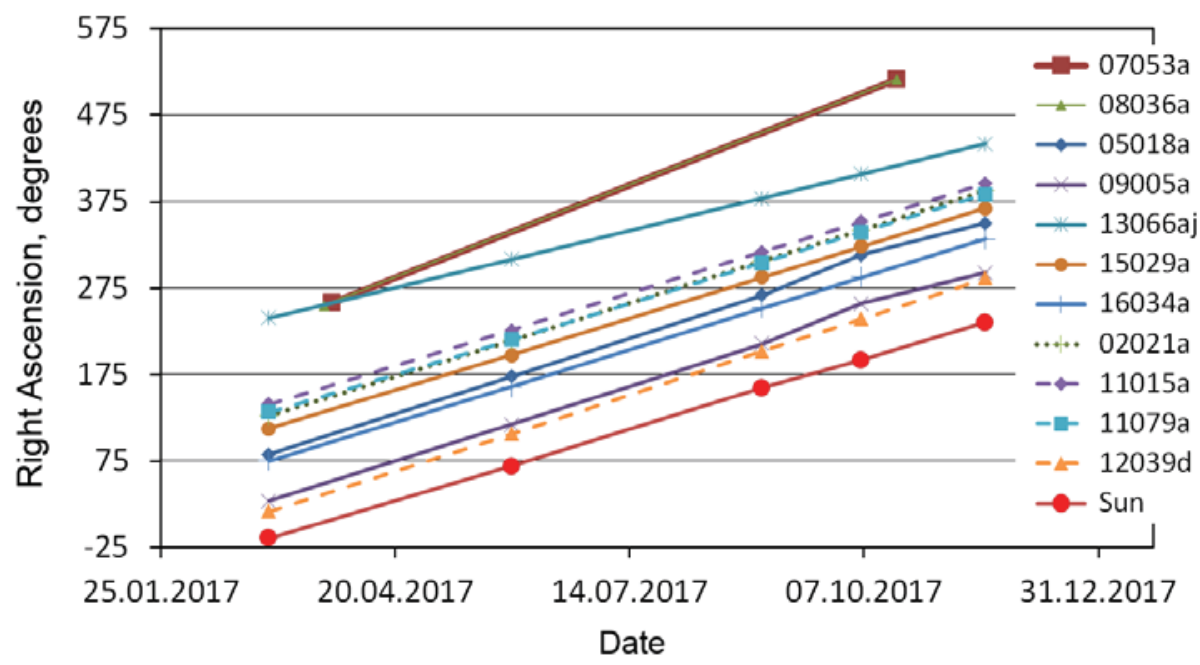

Fig. 5. Shifts of the orbital planes of the selected LEO objects with respect to the Sun in 2017 


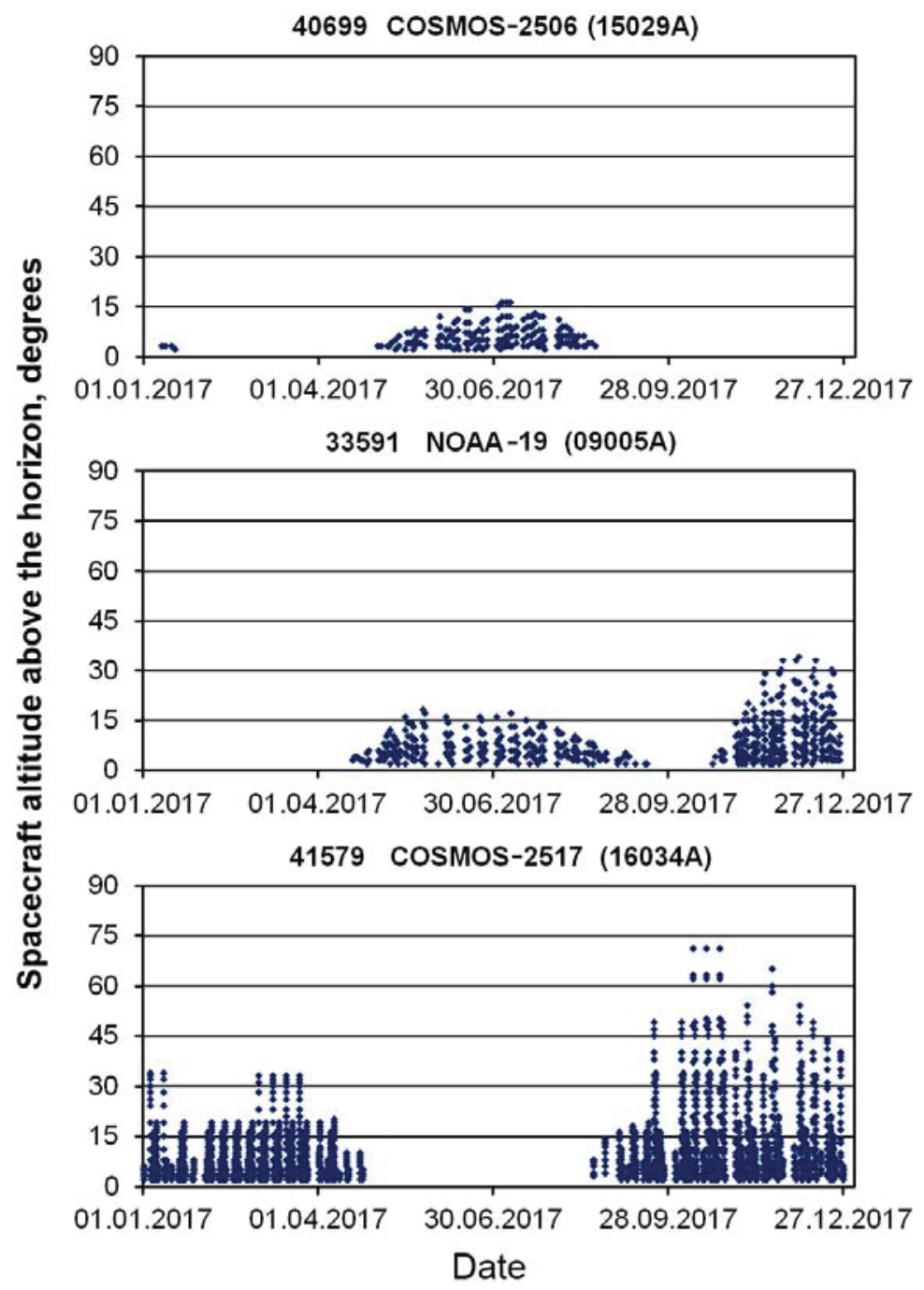

Fig. 6. Visibility of passes of the spacecrafts COSMOS-2506 (upper panel), NOAA-19 (middle panel) and COSMOS-2517 (bottom panel) during the year 2017 at the equatorial OSF plotted as variations in the spacecraft altitude above the horizon

The altitudes above the horizon for all visible passes of the spacecraft NOAA-19 (09005A) are shown in Figure 6 (middle panel). As in the earlier described case, the altitudes at culmination for two intervals of the spacecraft visibility in 2017 did not exceed $17^{\circ}$ in summer season and $35^{\circ}$ in the later part of the year. Angle D for the spacecraft's orbit during this period varied within the range of $43^{\circ}-65^{\circ}$.

For the spacecraft Cosmos-2517 (16034A) shown in Figure 6 (bottom panel) with $90^{\circ}$ angle between its orbital ascending node and the Sun, the conditions of visibility at the equatorial OSF are far more favourable. Over the two intervals of the spacecraft visibility, the altitude at culmination reached $34^{\circ}$ and $70^{\circ}$, respectively.

The following results were obtained for the other target LEO spacecrafts. Four of them, namely, Spot-5 (02021A), ZY-1 (11079A), ResourceSat-2 (11015A) and TET-1 (12039A) had no passes visible at the equatorial OSF. The angular distance between the orbits of the first three mentioned spacecrafts and the Sun was about $147^{\circ}, 152^{\circ}$ and $161^{\circ}$, respectively, while the angular distance between the orbit of the latter spacecraft and the Sun was $31^{\circ}-51^{\circ}$. In 2017, there were intervals of good visibility of NOAA-18 (05018A) and BPA-3 
(13066AJ), as well as SAR-Lupe 3 and SAR-Lupe 5. The orbital planes of the last three LEO spacecrafts drifted noticeably with respect to the Sun.

Thus, we have illustrated that the conditions of visibility of LEO spacecrafts in SSO at the equatorial OSF depend primarily on the orientation of their orbital planes with respect to the Sun.

The results obtained were additionally verified by calculating 1-year-ahead conditions of visibility of the indicated spacecrafts. When conducting these calculations, we computed changes in the orbital elements using the SGP4 model of the satellite motion in the Earth's gravitational field to employ them in preference to the actual changes in the orbital elements recorded over that year (based on the current data in the CSpOC/USStratCom catalogue). We carried out calculations of the position of these LEO objects, and we can state that in the case of a long-term prediction (1 year) and in the case of frequently updated orbit elements, the differences in the maximal height of path above the horizon (culmination height) reach several degrees (maximum up to $20^{\circ}$ for near-zenith passage). Significant errors in the position of the objects are observed primarily along the trajectory (up to 3-5 minutes in time). These estimates allow us to qualitatively predict the visibility of the LEO objects' trajectory, since these prediction errors cannot affect the general conclusions regarding the observability of these objects. These calculations confirmed the absence of visible passes for the four spacecrafts mentioned above, as well as for two spacecrafts with low altitudes at culmination, namely, Cosmos-2506 and NOAA-19.

In order to confirm the pattern of observability (visibility) of the passes of LEO spacecrafts in SSO through the FOV of the equatorial OSF, we selected 16 more LEO spacecrafts in SSO with orbital inclinations $98^{\circ}-99^{\circ}$ and a mean motion within the range of 13.4-15.3 revolutions per day (Table 2). The spacecrafts were selected in such a manner as to ensure that in 2017 , angle $\mathrm{D}$ would be close to $0^{\circ}, 90^{\circ}, 180^{\circ}$ and $270^{\circ}$, respectively, for each foursome (Figure 7).

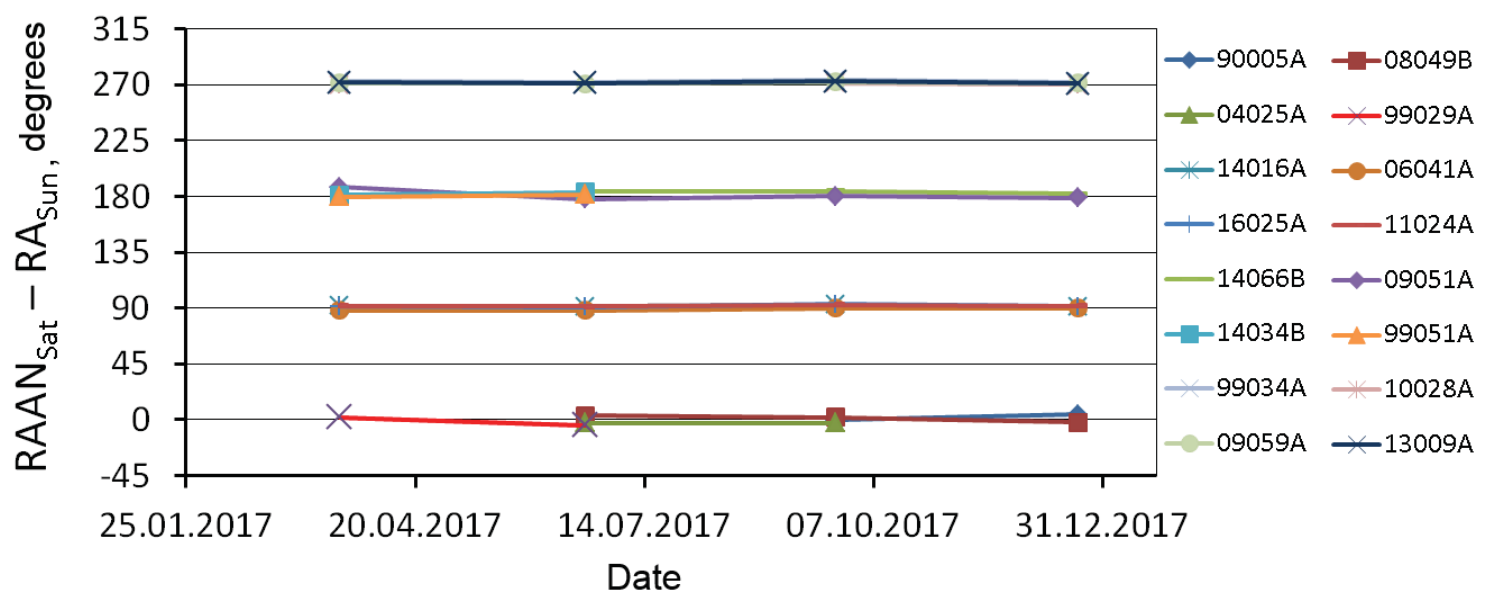

Fig. 7. The difference between the right ascension of the ascending node of the orbits of 16 LEO spacecrafts in SSO in 2017 and the relevant right ascension of the Sun (angle D) 
Table 2. LEO objects in Sun-synchronous orbits, selected for the simulation of the observability at the equatorial OSF.

\begin{tabular}{|c|l|}
\hline $\begin{array}{c}\text { Reference no. } \\
\text { in the CSpOC / USStratCom } \\
\text { catalogue }\end{array}$ & \multicolumn{1}{|c|}{$\begin{array}{c}\text { Name of the LEO object } \\
\text { (International Identification } \\
\text { Number) }\end{array}$} \\
\hline 20436 & Spot-2 (90005A) \\
\hline 25756 & KitSat-3 (99029A) \\
\hline 25789 & Quikscat (99034A) \\
\hline 25919 & Iconos-2 (99051A) \\
\hline 28366 & ApprizeSat-2 (04025A) \\
\hline 29479 & Hinode (06041A) \\
\hline 33397 & SL-24 (08049B) \\
\hline 35931 & OceanSat-2 (09051A) \\
\hline 36036 & SMOS (09059A) \\
\hline 36598 & Picard (10028A) \\
\hline 37673 & Sac-D (11024A) \\
\hline 39086 & Saral (13009A) \\
\hline 39634 & Sentinel-1a (14016A) \\
\hline 40054 & Aisat (14034B) \\
\hline 40287 & CZ-2c R/B (14066B) \\
\hline 41456 & Sentinel-1b (16025A) \\
\hline
\end{tabular}

The visibility of the listed LEO spacecrafts at the equatorial OSF during 2017 was calculated. It turned out that there were no visible passes in 2017 for all LEO spacecrafts with $\mathrm{D}$ close to $0^{\circ}$ or $180^{\circ}$. The other spacecrafts (with D close to $90^{\circ}$ or $270^{\circ}$ ) were visible for much of 2017.

The visibility of the Aquarius spacecraft (37673, 11024A), for which angle D is close to $90^{\circ}$, as per prediction for 2017 is shown in Figure 8 (upper panel). For each pass, there is only one point plotted on the $\mathrm{Y}$-axis corresponding to the altitude at culmination at the visible part of the orbit (these points are connected in a solid line just to underscore the periodic pattern of visibility of the satellite passes). Passes with altitudes at culmination less than $10^{\circ}$ are not shown. As can be seen in the figure, this spacecraft could be observed in 2017 rather regularly during morning and evening twilight. 


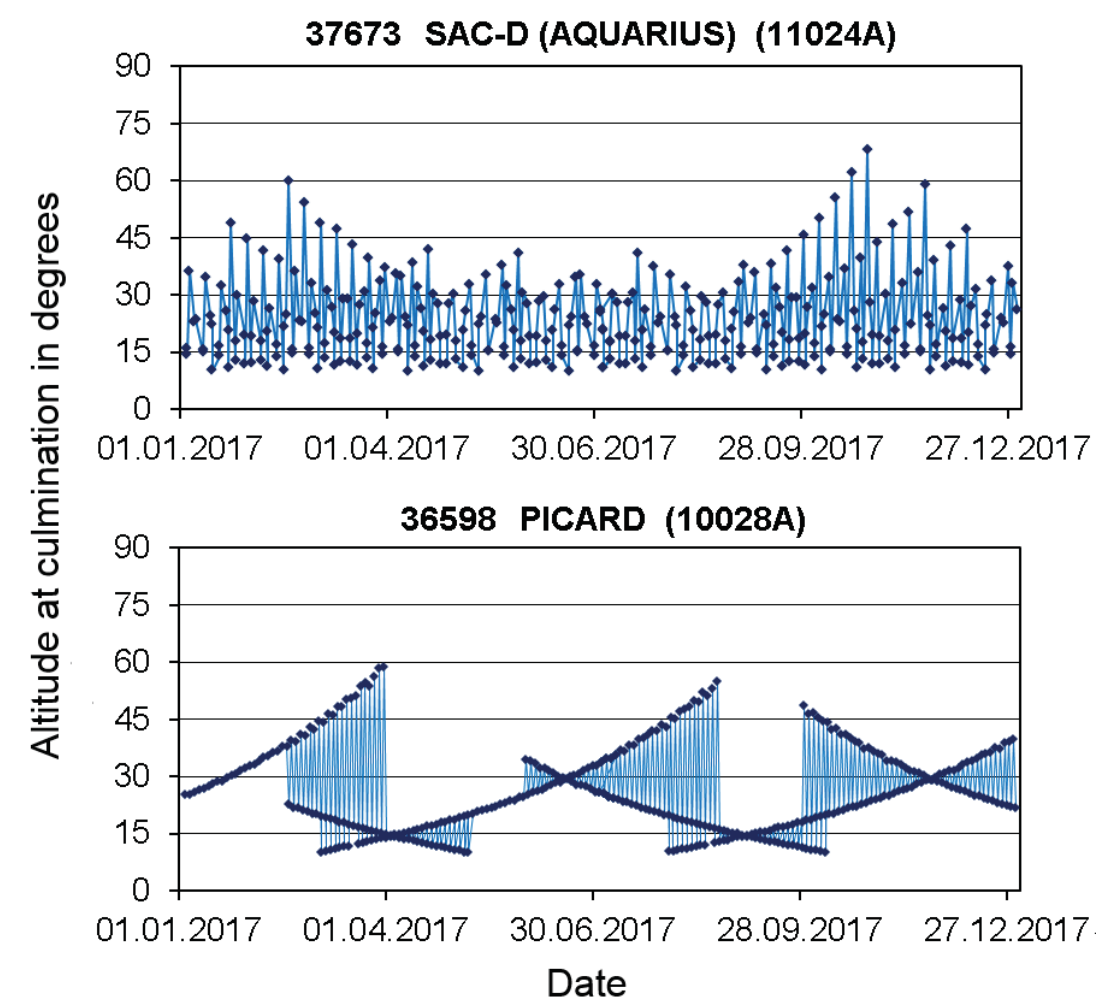

Fig. 8. The altitudes at culmination of the passes of the spacecrafts Aquarius 37673 (upper panel) and Picard 36598 (lower panel) observed at the equatorial OSF in 2017

A different pattern of visibility has been exhibited by the Picard spacecraft 36598 (Figure 8 , lower panel) for which angle $\mathrm{D}$ is close to $270^{\circ}$; in particular, the satellite's path lies in the vicinity of terminator, and hence, it can also be seen only during evening or morning twilight. However, the satellite was visible virtually throughout the year 2017 (as there were always visible passes culminating at altitudes higher than $15^{\circ}$ ).

It can be summarized that for circular SSO, the visibility of LEO spacecrafts at the OSF located on the equator is determined primarily by the orientation of their orbital planes with respect to the Sun: if the difference between the RAAN of the LEO spacecraft orbit and relevant right ascension of the Sun is close to $0^{\circ}$ or $180^{\circ}$, then there are no passes of the LEO spacecraft through the FOV of the equatorial OSF, which could be suitable for optical observations; if this difference is approaching $90^{\circ}$ or $270^{\circ}$, then there are intervals of different lengths during a year when the passes culminate at an altitude sufficient for observations. The pattern of such "visibility" of passes is determined by the D value (i.e., orientation of the orbital plane relative to the Sun) and not obviously dependent on the length of the semi-major axis, inclination or eccentricity of a given LEO spacecraft orbit.

The afore-described calculations were conducted for the OSF located on the equator at one fixed longitude. For any other equatorial OSF with a longitude that differs from our accepted value by an angle which is a multiple of the orbital period of the observed LEO spacecraft $\left(\Delta \lambda^{\circ}\right.$ $=$ Porbit $/ 4$ in minutes), the visibility conditions will be repeated in exactly the same way (for the target SSO, this angle $\Delta \lambda^{\circ}$ is approximately equal to $25^{\circ}$ ). For any equatorial OSF with a longitude that differs from our accepted value by an intermediate angle (which is not a multiple of $\Delta \lambda^{\circ}$ ), the conditions of a spacecraft's visibility will be different as it will be seen at another altitude above the horizon at a slightly different local solar time. However, as the conditions of illumination of a spacecraft by the Sun do not depend on an observer's location, a few days after the orbital plane shift (along with the Sun), the above-specified visibility conditions become true for the given OSF. The conditions of spacecraft visibility at any other equatorial 
OSF will not change fundamentally. It is borne out by the equivalent number of LEO objects and their passes visible at three different equatorial OSF as shown in Figure 4.

\section{TIME CHARACTERISTICS OF PASSES OF LEO OBJECTS THROUGH THE FOV OF THREE OSF}

The temporal frequency of passes of LEO objects into the OSF FOV and duration of their observation while passing through the FOV can be considered as the key time characteristics of the LEO object passes. The results of simulation of these characteristics are presented in Tables 3 and 4. Note that such results were obtained leaving out the LEO objects, for which the duration of passing through the FOV of the relevant OSF was less than a minute, as observations involving the tracking over such short time spans were not meaningful. Therefore, the total frequency of passes is slightly greater while the mean duration of passing through the FOVs is slightly shorter than those given in the tables.

Table 3. Time characteristics of the passes of LEO objects through the fields of view of three OSF depending on the time of year

\begin{tabular}{|c|c|c|c|c|c|c|c|c|c|}
\hline \multirow{3}{*}{ Date } & \multicolumn{3}{|c|}{ OSF-1 } & \multicolumn{3}{|c|}{ OSF-2 } & \multicolumn{3}{|c|}{ OSF-3 } \\
\hline & \multicolumn{2}{|c|}{$\begin{array}{l}\text { Frequency of } \\
\text { passes into the } \\
\text { field of view, } \\
\text { number of } \\
\text { passes per hour }\end{array}$} & \multirow{2}{*}{$\begin{array}{l}\text { Mean } \\
\text { duration of } \\
\text { passing } \\
\text { through the } \\
\text { field of } \\
\text { view, } \\
\text { minutes }\end{array}$} & \multicolumn{2}{|c|}{$\begin{array}{l}\text { Frequency of } \\
\text { passes into the } \\
\text { field of view, } \\
\text { number of } \\
\text { passes per hour }\end{array}$} & \multirow{2}{*}{$\begin{array}{l}\text { Mean } \\
\text { duration of } \\
\text { passing } \\
\text { through the } \\
\text { field of } \\
\text { view, } \\
\text { minutes }\end{array}$} & \multicolumn{2}{|c|}{$\begin{array}{l}\text { Frequency of } \\
\text { passes into the } \\
\text { field of view, } \\
\text { number of } \\
\text { passes per hour }\end{array}$} & \multirow{2}{*}{$\begin{array}{l}\text { Mean } \\
\text { duration of } \\
\text { passing } \\
\text { through the } \\
\text { field of } \\
\text { view, } \\
\text { minutes }\end{array}$} \\
\hline & Min. & Max. & & Min. & Max. & & Min. & Max. & \\
\hline $\begin{array}{l}\text { Vernal } \\
\text { equinox }\end{array}$ & 1010 & 2355 & 7.03 & 0 & 1500 & 8.03 & 3350 & 4150 & 7.6 \\
\hline $\begin{array}{l}\text { Summer } \\
\text { solstice }\end{array}$ & 1650 & 2250 & 8.63 & 0 & 1470 & 7.29 & 700 & 3075 & 7.38 \\
\hline $\begin{array}{l}\text { Autumn } \\
\text { equinox }\end{array}$ & 920 & 2250 & 7.09 & 0 & 1350 & 8.21 & 3475 & 4200 & 8.62 \\
\hline $\begin{array}{l}\text { Winter } \\
\text { solstice }\end{array}$ & 2 & 2200 & 7.6 & 0 & 1500 & 7.55 & 3700 & 4100 & 9.13 \\
\hline
\end{tabular}

Table 4. Time characteristics of LEO spacecrafts passing through the fields of view of three OSF depending on the time of year.

\begin{tabular}{|c|c|c|c|c|c|c|c|c|c|}
\hline \multirow{3}{*}{ Date } & \multicolumn{3}{|c|}{ OSF-1 } & \multicolumn{3}{|c|}{ OSF-2 } & \multicolumn{3}{|c|}{ OSF-3 } \\
\hline & \multicolumn{2}{|c|}{$\begin{array}{l}\text { Frequency of } \\
\text { passes into the } \\
\text { field of view, } \\
\text { number of } \\
\text { passes per hour }\end{array}$} & \multirow{2}{*}{$\begin{array}{l}\text { Mean } \\
\text { duration of } \\
\text { passing } \\
\text { through the } \\
\text { field of } \\
\text { view, } \\
\text { minutes }\end{array}$} & \multicolumn{2}{|c|}{$\begin{array}{l}\text { Frequency of } \\
\text { passes into the } \\
\text { field of view, } \\
\text { number of } \\
\text { passes per hour }\end{array}$} & \multirow{2}{*}{$\begin{array}{l}\text { Mean } \\
\text { duration of } \\
\text { passing } \\
\text { through the } \\
\text { field of } \\
\text { view, } \\
\text { minutes }\end{array}$} & \multicolumn{2}{|c|}{$\begin{array}{l}\text { Frequency of } \\
\text { passes into the } \\
\text { field of view, } \\
\text { number of } \\
\text { passes per hour }\end{array}$} & \multirow{2}{*}{$\begin{array}{l}\text { Mean } \\
\text { duration of } \\
\text { passing } \\
\text { through the } \\
\text { field of } \\
\text { view, } \\
\text { minutes }\end{array}$} \\
\hline & Min. & Max. & & Min. & Max. & & Min. & Max. & \\
\hline $\begin{array}{l}\text { Vernal } \\
\text { equinox }\end{array}$ & 10 & 240 & 5.57 & 0 & 130 & 6.75 & 170 & 380 & 5.16 \\
\hline $\begin{array}{l}\text { Summer } \\
\text { solstice }\end{array}$ & 70 & 290 & 7.61 & 0 & 110 & 6.63 & 15 & 210 & 6.97 \\
\hline $\begin{array}{l}\text { Autumn } \\
\text { equinox }\end{array}$ & 10 & 140 & 6.53 & 0 & 120 & 8.4 & 170 & 415 & 5.66 \\
\hline $\begin{array}{l}\text { Winter } \\
\text { solstice }\end{array}$ & 0 & 165 & 7.7 & 0 & 130 & 6.9 & 260 & 400 & 8.1 \\
\hline
\end{tabular}

Based on these calculations, in Figure 9, we have plotted variations in the limiting values (maximal and minimal) of the mean frequency of passes of LEO objects observed at three selected OSF at different times of the year. As can be seen, the frequency of passes is 
maximal for the sub-polar OSF-3. Meanwhile, the southern OSF shows a noticeable minimum of the frequency of passes of LEO objects in June, as for this OSF, the Earth's shadow rises as high as possible above the horizon. At the same time, the mean frequency of passes of LEO objects for the northern mid-latitude OSF-1 is maximising, although it is usually 1.5-2 times lower than that for the sub-polar OSF throughout a year. The lowest mean frequency of passes can be observed for the equatorial OSF-2. Also, while the maximum mean overnight frequency of passes for this OSF is virtually constant throughout a year, being about $\sim 1,500$ passes of LEO objects per hour, the minimum one always reaches zero.

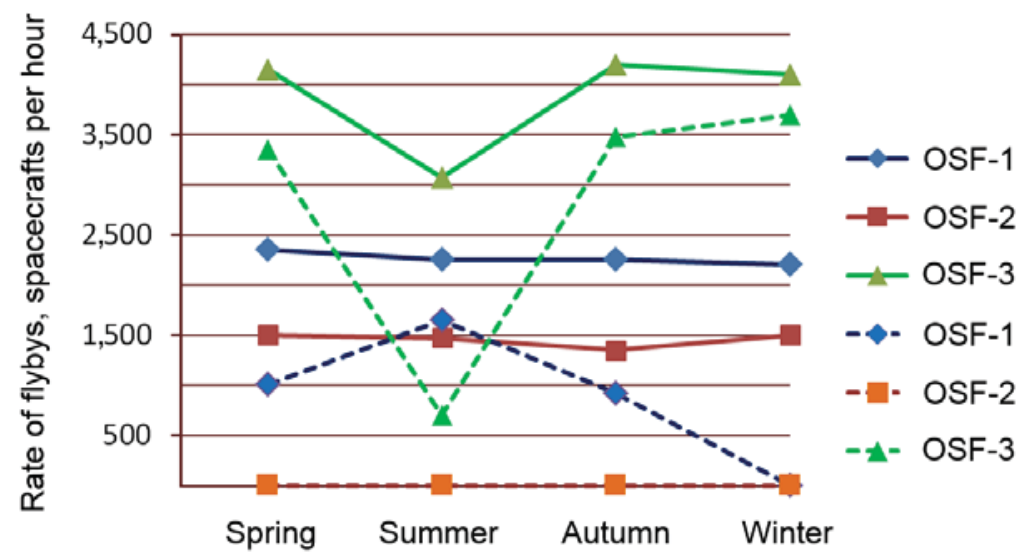

Fig. 9. The mean overnight frequency of LEO object passes over three OSF at different times of the year (the maximal frequencies are shown as solid lines and the minimal ones as dotted lines)

Variations in the overnight frequency of passes for each OSF are illustrated in Figures 10 12. As can be seen in these figures, the flux of the potentially observable LEO objects (and LEO spacecrafts, respectively) through the FOVs is not steady; it varies over a single night and changes depending on the time of year. It should be noted that for the equatorial OSF-2, there was always a period of zero visibility in the dead of night when LEO objects could not be observed at all. This is primarily due to the fact that objects in low orbits are in the Earth's shadow. The presence of noticeable spikes of the plotted number of passing space objects corresponding to the first bars (first time intervals) on virtually all diagrams for LEO objects can be associated with the following: when a shadow falls over the observation point, all space objects within the FOV immediately become visible, including those which have just passed into the FOV and those that have already been passing through the FOV and remained invisible until the shadow was cast over the observation facility. These first "anomalous" bars (intervals) were not factored in calculating the data presented in Tables 3 and 4. 

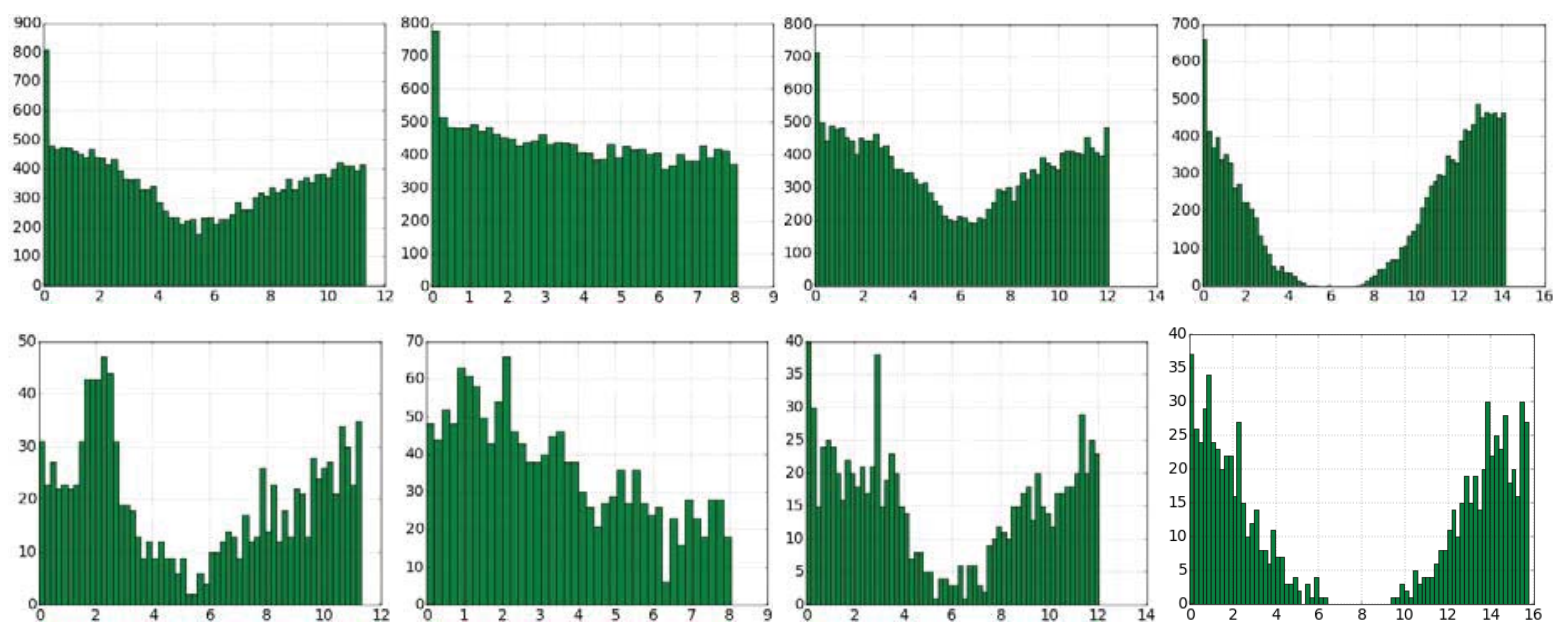

Fig. 10. Overnight variations in the frequency of passes of LEO objects (upper panel) and LEO spacecrafts (lower panel) through the OSF-1 field of view with 0.2-hour intervals: left to right, the vernal equinox, summer solstice, autumn equinox and winter solstice. The time is expressed in hours from the observation start
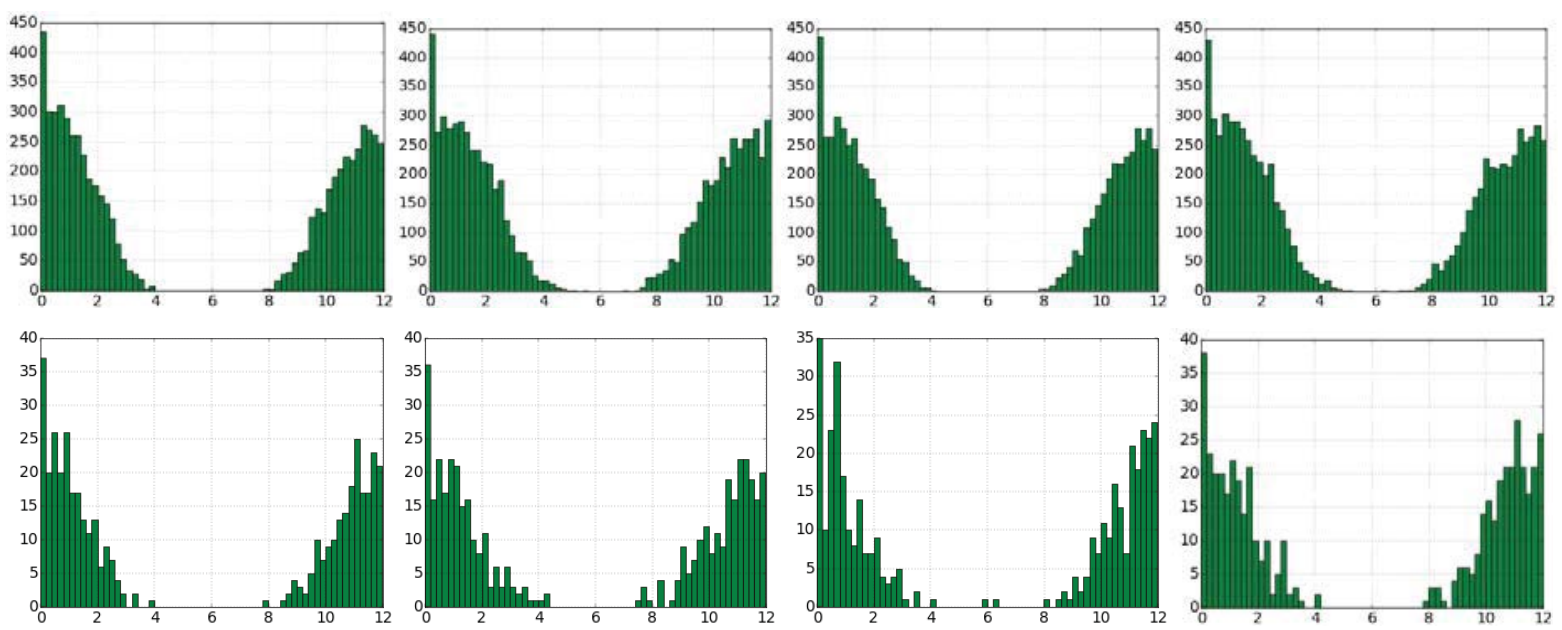

Fig. 11. Overnight variations in the frequency of passes of LEO objects (upper panel) and LEO spacecrafts (lower panel) through the equatorial OSF-2 field of view with 0.2-hour intervals: left to right, the vernal equinox, summer solstice, autumn equinox and winter solstice. The time is expressed in hours from the observation start 

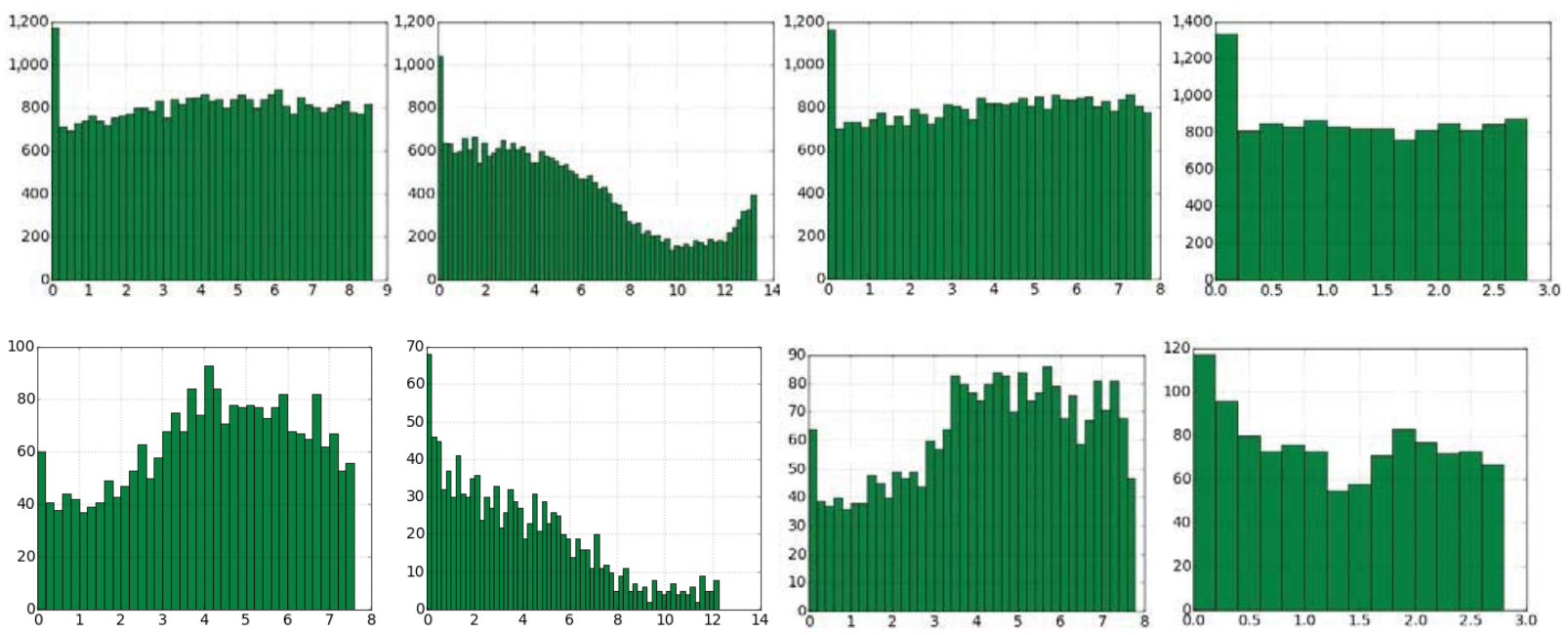

Fig. 12. Overnight variations in the frequency of passes of LEO objects (upper panel) and

LEO spacecrafts (lower panel) through the field of view of the southern subpolar OSF-3 with

0.2-hour intervals: left to right, the vernal equinox, summer solstice, autumn equinox and winter solstice. The time is expressed in hours from the observation start

\section{CONCLUSION}

1. The following have been deduced from the results of the simulation modelling of the LEO object passing through the FOV of OSF with different geographic locations:

- The number and temporal frequency of visible passes of LEO objects through the OSF FOV can vary significantly both over a year and a single night of observations.

- The OSF located on the equator is substantially less capable of observing LEO objects, especially those in SSO, due to specific aspects of their lighting conditions.

2. The techniques and results reported in this paper can be further used in the location planning of new OSF to enable them to observe the whole set of LEO objects.

3. In future research, it would be reasonable to focus more on a thorough analysis of the visibility conditions of LEO objects, especially those in SSO.

\section{REFERENCES}

Chandana Peiris T. (2017) Challenges of Space Debris and Site Selection Criteria to Install Optical Telescope to Observe Space Debris in Sri Lanka, Proc. of the 15th Open University Research Sessions (OURS 2017), The Open University of Sri Lanka, 527-531.

Friedman A.M., Frueh C. (2018) Determining Characteristics of Artificial Near-Earth Objects Using Observability Analysis, Acta Astronautica, Vol. 144, 405-421; (https://doi.org/10.1016/j.actaastro.2017.12.028).

Gasdia F., Barjatya A., Bilard S. (2017) Multi-Site Simultaneous Time-Resolved Photometry with a Low Cost Electro-Optics System, Sensors 2017, 17, 1239; doi:10.3390/s17061239 (www.mdpi.com/journal/sensors)

Hoots F.R., Roehrich R.L. (1980) Models for Propagation of NORAD Element Sets, Spacetrack Report, No. 3, Colorado Springs: Peterson AFB, CO, 91 p. Available online at http://www.celestrak.com/NORAD/documentation/spacetrk.pdf. 
Koshkin N., Shakun L., Korobeynikova E., Melikyants S., Strakhova S., Dragomiretsky V., Ryabov A., Golubovskaya T., Terpan S. (2018) Monitoring of Space Debris Rotation Based on Photometry, Odessa Astron. Publ., Vol. 31, 179-185; (https://doi.org/10.18524/1810-4215.2018.31.147807).

Lal B., Balakrishnan A. et al. (2018). Global Trends in Space Situational Awareness (SSA) and Space Traffic Management (STM), IDA Science \& Technology Policy Institute, Available online at https://www.ida.org/idamedia/Corporate/Files/Publications/STPIPubs/2018/D-9074.pdf

Mike McCants' Satellite Tracking TLE ZIP Files. The classified elements zip file. Available online at http://www.prismnet.com/ mmccants/tles/classfd.zip

NORAD Two-Line Element Set Format. Available online at http://celestrak.com/NORAD/documentation/tle-fmt.asp

Piergentili F., Ceruti A., Rizzitelli F., Cardona T., Battagliere M.L., Santoni F. (2014). Space Debris Measurement Using Joint Mid-Latitude and Equatorial Optical Observations, IEEE Transactions on Aerospace and Electronic Systems, Vol. 50, No. 1, 664-675.

Sánchez-Ortiz N., Torres J.N., Domínguez-González R., López N.G. (2018) Accurate Optical Observation of Space Objects in LEO regime, 2018 Advanced Maui Optical and Space Surveillance Technologies Conference.

Schildknecht T., Hinze A., P. Schlatter, J. Silha, J. Peltonen, T. Säntti, T. Flohrer (2014) Improved Space Object Orbit Determination Using CMOS Detectors, 2014 Advanced Maui Optical and Space Surveillance Technologies Conference.

Shakun L.S., Koshkin N.I. (2014) Determination of Visible Coordinates of the Low-Orbit Space Objects and Their Photometry by the CCD Camera with the Analogue Output. Initial Image Processing, Advances in Space Research, 2014, Vol. 53, No. 12, 1834-1847; (http://doi.org/10.1016/j.asr.2013.12.009).

Shakun L., Korobeynikova E., Koshkin N., Melikyants S., Strakhova S., Terpan S., Burlak N., Golubovskaya T., Dragomiretsky V., Ryabov A. (2016) The observations of Artificial Satellites and Space Debris Using KT-50 Telescope in the Odessa University, Odessa Astron. Publ., Vol. 29, 217-220; (http://dx.doi.org/10.18524/1810-4215.2016.29.85234).

Shakun L., Koshkin N., Korobeynikova E., Strakhova S., Dragomiretsky V., Ryabov A., Melikyants S., Golubovskaya T., Terpan S. (2017) Accuracy of Satellite Optical Observations and Precise Orbit Determination, Odessa Astron. Publ., Vol. 30, 242-245; (http://dx.doi.org/10.18524/1810-4215.2017.30.117660).

Silha J., Pittet J.-N., Hamara M., Schildknecht Th. (2018) Apparent Rotation Properties of Space Debris Extracted from Photometric Measurements, Advances in Space Research, 2018, Vol. 61, No. 3, 844-861; (https://doi.org/10.1016/j.asr.2017.10.048). 
SSA Sharing \& Orbital Data Requests (ODR). Available online at https://www.spacetrack.org/documentation\#odr

Vananti A., Schildknecht T., Pinna G.M., Flohrer T. (2013) Assessment of possible observation strategy in LEO regime, Proc. 64 ${ }^{\text {th }}$ International Astronautical Congress (2327 September 2013, Beijing, China).

Received: 2019-08-02,

Reviewed: 2019-10-10 and 2019-10-15,

Accepted: 2019-12-11. 